Null models are used to assess departures from randomness in other fields such as palaeobiology ${ }^{10}$ and the reconstruction of phylogeny ${ }^{11}$, so this new analytical procedure will be useful beyond community ecology. Some interlocking statistical and biological concerns remain, however, centred on the problem of interdependencies.

For example, in the Vanuatu analysis pairs occurring nine and only nine times, like the twos, are improbably rare, but pairs occurring ten and only ten times are improbably common. These opposite directions in deviation of 'neighbours' in the frequency distribution (and there are others) hint at further structure in the data, structure that may be statistical ${ }^{12,13}$ or biological. The source of that structure might lie in the full set of 56 bird species being analysed together, rather than just guilds of potential competitors, and in the artificiality (and convenience) of holding the number of islands per species constant, for which there is no biological theory; in contrast there is good biogeographical theory ${ }^{14}$ to justify holding the number of species per island constant in the null modelling.

This raises the issue of how a biologically realistic null model can be constructed for communities of species in an archipelago when the source area and set of species cannot be specified; and of how sequential colonization and local adaptation, geography and history ${ }^{15}$, can be adequately built into the null model. In fact, how much biology should be incorporated into the null model?

Here we have echoes of a major issue in population genetics - whether variation is maintained by random drift or selection. We are left, as Diamond ${ }^{2}$ was, with a challenging problem, that of determining the relative influences of chance and necessity (systematic forces, such as competition) in the assembly of biological communities through time.

Peter R. Grant is in the Department of Ecology and Evolutionary Biology, Princeton University,

Princeton, New Jersey, 08544-1003, USA.

e-mail:prgrant@princeton.edu

1. MacArthur, R. H. Geographical Ecology (Princeton Univ. Press, 1972).

2. Diamond, J. in Ecology and Evolution of Communities (eds Cody, M. L. \& Diamond, J. M.) (Harvard Univ. Press, 1975).

3. Sanderson, J. G., Moulton, M. P. \& Selfridge, R. G. Oecologia 116, 275-283 (1998).

4. Connor, E. F. \& Simberloff, D. Ecology 60, 1132-1140 (1979).

5. Wiens, J. A. The Ecology of Bird Communities, Vol. 1 (Cambridge Univ. Press, 1989).

6. Gotelli, N. J. \& Graves, G. R. Null Models in Ecology (Smithsonian Press, Washington, DC, 1996).

7. Grant, P. R. \& Abbott, I. Evolution 34, 332-341 (1980)

8. Colwell, R. K. \& Winkler, D. W. in Ecological Communities (eds Strong, D. R. Jr, Simberloff, D., Abele, L. G. \& Thistle, A. B.) (Princeton Univ. Press, 1984).

9. Harvey, P. H., Colwell, R. K., Silvertown, J. W. \& May, R. M. Annu. Rev. Ecol. Syst. 14, 189-211 (1985).

10. Raup, D. M., Gould, S. J., Schopf, T. J. M. \& Simberloff, D. S, J. Geol. 81, 525-542 (1973)

11. Harvey, P. H., Brown, A. J. L., Maynard Smith, J. M. \& Nee, S. (eds) New Uses for New Phylogenies (Oxford Univ. Press, 1996). 12. Roberts, A. \& Stone, L. Oecologia 83, 560-567 (1990).

13. Stone, L. \& Roberts, A. Oecologia 91, 419-424 (1992).

14. MacArthur, R. H. \& Wilson, E. O. The Theory of Island Biogeography (Princeton Univ. Press, 1967).

15. Ricklefs, R. E. \& Schluter, D. Species Diversity in Ecological Communities (Univ. Chicago Press, 1996).

\title{
An atomic dimmer switch
}

\author{
Philip H. Bucksbaum
}

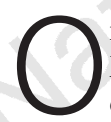
n page 239 of this issue ${ }^{1}$, Doron Meshulach and Yaron Silberberg of the Weizmann Institute show how atoms can be made to absorb light more or less readily, or not at all, simply by controlling the phase of light shone at them. This joins a growing list of new 'coherent control' methods for manipulating the internal quantum dynamics of atoms and molecules using the coherence properties of light, rather than its intensity or colour.

All atoms and molecules can absorb light. The absorption usually occurs for light that has a frequency $\nu$ at resonance - that is where the energy of a single photon in the light beam, $h \nu$, equals the energy difference between the ground state and an excited quantum state of the system. But when an atom is subjected to intense light, such as that produced by a laser, nonlinear absorption is also possible. In nonlinear absorption, two or more photons pool their energy to excite the atom, and the sum of the photon energies equals the excited-state energy difference. The photons must not only have the right

NATURE|VOL 396 | 19 NOVEMBER 1998 | www.nature.com total energy, they must also arrive at the target at nearly the same time, so nonlinear optical effects are usually stronger when the light is compressed into a short, intense pulse. This is the principle behind several practical devices in lasers such as harmonic frequency converters.

One reason that the arrangement of photons is important in a nonlinear process is quantum interference. When a quantum dynamical process can take more than one path, interference occurs between the different possible routes. The interference may be destructive or constructive, slowing or has-

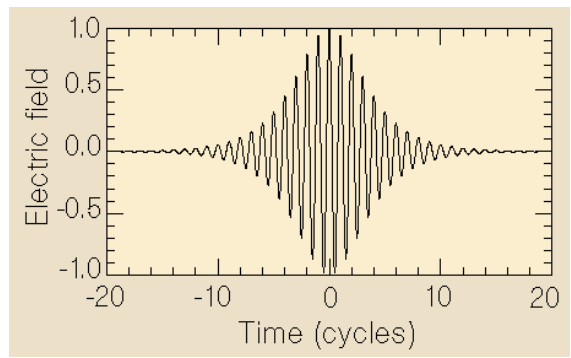

Figure 1 The electric field of a short laser pulse. tening the process. An example is the twophoton absorption experiment described by Meshulach and Silberberg: if the photons are both present at the same time, then the absorption may take two paths, corresponding to absorption of photon 1 first, followed by photon 2, or the other way around. Coherent control provides a way to adjust the quantum interferences between these different paths, affecting the rate of the whole process.

This is easier to understand if we stop talking about photons and consider the light as a classical electromagnetic wave. The light pulse is a travelling wave with frequency $\nu$ and electric field amplitude $A$, so that an atom will experience the light as an oscillating electric field $E=A \cos (2 \pi \nu t)$ where $t$ is time. Figure 1 is a picture of the electric field versus time for a typical short laser pulse. The turn-on and turn-off create a frequency spread, shown in the spectral density of this pulse given by its Fourier transform (Fig. 2a).

An atom with a resonance at any frequency in this spectrum can be excited by absorbing a single photon of light from the pulse. Each frequency present can have different phases as well as different amplitudes - that is, the light with frequency $\nu_{1}$ might be a sine wave, $\sin \left(2 \pi \nu_{1} t\right)$ while the light with $\nu_{2}$ might be a cosine wave $\cos \left(2 \pi \nu_{2} t\right)$ or any intermediate phase. In Fig. 1, all the different frequencies happen to be cosine waves.
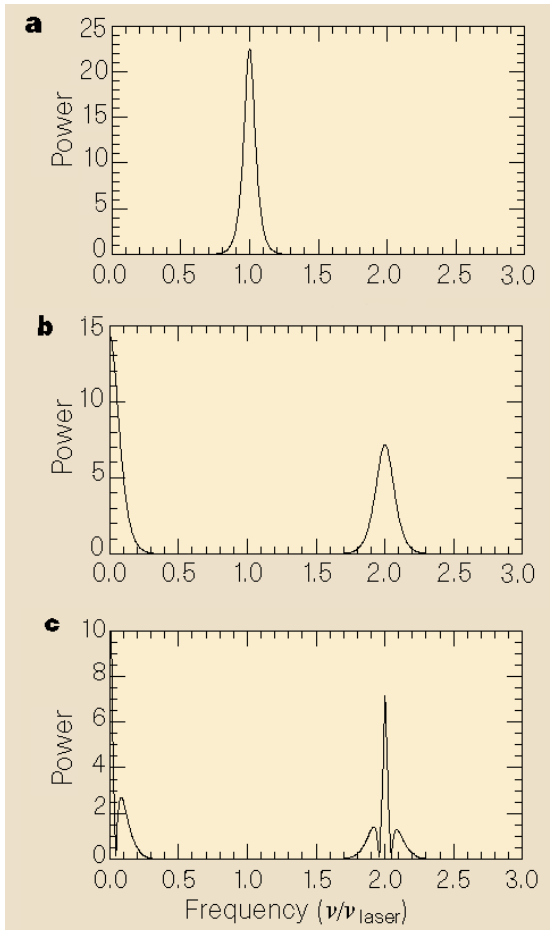

Figure 2 Spectral power distributions. a, The spectrum of the pulse in Fig. 1. b, The twophoton spectrum of the same pulse. $c$, The twophoton spectrum of a similar pulse, whose spectral components with frequency greater than the mean have been reversed in sign. The two-photon nonlinear absorption rate is sharpened. 


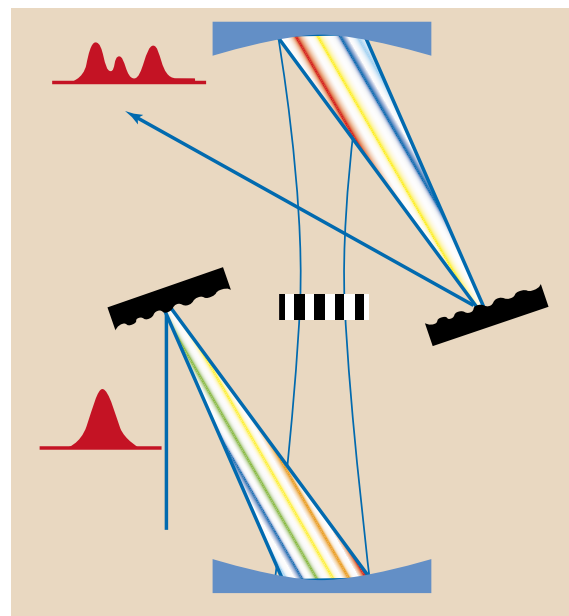

Figure 3 A pulse shaper, capable of imposing selective phase changes on a laser pulse.

Now if there is no resonance at the principal frequency, but one at twice that frequency, then absorption only occurs for pairs of photons. The atom no longer responds resonantly to the applied electric field, but rather to the square of the field $E^{2}(t)=A^{2}(t) \cos ^{2}(2 \pi \nu t)$, (the probability of a single photon's presence is proportional to the field, so the probability of two being present at once is proportional to the square). That has a completely different frequency spectrum (Fig. 2b), one peak of which happens to be at twice the laser frequency - that is at the position of the nonlinear resonance for two-photon absorption.

If one now adjusts the various phases of the different colours (or frequencies) in the initial spectrum, but leaves all of their amplitudes just the same, then the spectral energy density of the light field $E(t)$ stays the same, but the spectral density of the nonlinear field shown in Fig. $2 \mathrm{~b}$ can change. For example, let's reverse the sign of the upper half of the spectrum in Fig. 2a; in other words, transform the spectral components above the laser frequency from cosine waves to minus cosine waves. The power spectrum of $E^{2}(t)$ now looks like Fig. 2c. By manipulating phases, the nonlinear spectral density can change so much that any particular resonant frequency in the spectrum can be made to vanish, and absorption at that frequency will vanish as well.

To achieve this degree of control, the Weizmann group made use of a new tool in optics called a pulse shaper ${ }^{2}$ (Fig. 3). It is essentially two spectrometers arranged back-to-back. The first spectrometer disperses the light into its various colours, which are displayed along the exit plane of the device. At this exit plane are a series of filters side-by-side, which can attenuate or delay separate segments of the pulse spectrum. Meshulach and Silberberg's filters were parts of a programmable liquid crystal display, not very different from the one used in laptop computers, which could adjust the phase delay of each frequency band. The second spectrometer then puts the separated colours back together again, and the result is a pulse with the same colours as before, but arranged with different relative phases and/or amplitudes.

To the original pulse the researchers added a frequency modulation that changes the phase, not the amplitude, of any frequency; but its effect on the nonlinear absorption spectrum was dramatic. As they describe in their paper, the two-photon resonance could even be made to vanish.

This dependence of quantum processes on the coherence of the driving radiation has already been used to control molecular dissociation and ionization pathways ${ }^{3}$, current in semiconductors ${ }^{4}$, and the direction of photoemission $^{5}$. Ultrafast pulse shapers, which make it possible to manufacture very complicated phase distributions, have been used in time-domain coherent control experiments, where the control is achieved by manipulating the internal motion of a molecule, to guide it to a particular set of final states ${ }^{6}$. Pulse shapers have also been used to shape quan- tum wave packets in atoms ${ }^{7}$. The new use of computer-controlled programmable pulse shapers in nonlinear interference experiments adds further capabilities to the field of coherent control. This could facilitate different chemical reactions in applications where laser chemistry is important, such as photolithography. A shaped nonlinear spectrum could also aid or inhibit atomic motion in molecules or in solids, where the energies of the two photons subtract rather than add (Raman interference).

Philip H. Bucksbaum is in the Department of

Physics, University of Michigan, Ann Arbor,

Michigan 48109-1120, USA.

e-mail:phb@umich.edu

1. Meshulach, D. \& Silberberg, Y. Nature 396, 239-242 (1998).

2. Weiner, A. M., Heritage, J. P. \& Kirschner, E. M. J. Opt. Soc. Am. B 5, 1563-1572 (1988).

3. Gordon, R. J. \& Rice, S. A. Annu. Rev. Phys. Chem. 48, 601-641 (1997).

4. Dupont, E., Corkum, P. B., Liu, H. C., Buchanan, M. \& Wasilewski, Z. R. Phys. Rev. Lett. 74, 3596-3599 (1995).

5. Yin, Y. Y., Elliott, D. S. \& Shehadeh, R. Chem. Phys. Lett. 241, 591-596 (1995).

6. Bardeen, C. J. et al. J. Phys. Chem. A 101, 3815-3822 (1997).

7. Weinacht, T. C., Ahn, J. \& Bucksbaum, P. H. Phys. Rev. Lett. 80 5508-5511 (1998).

\section{Developmental neurobiology \\ First class way to develop a brain}

\section{Jonathan Howard and lan Thompson}

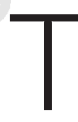
he major histocompatibility complex (MHC) is unambiguously part of the immune system. Generally speaking the immune system operates outside the blood-brain barrier, and this has been correlated with the belief that MHC glycoproteins are not expressed in the normal central nervous system. But this comforting correlation is now challenged by the radical observations of Carla Shatz and colleagues, published in Neuron ${ }^{1}$. They not only demonstrate, for the first time, considerable amounts of surfaceexpressed MHC glycoproteins in the optic system, but they also show that these proteins may be involved there in synaptic remodelling during development.

Shatz and co-workers were searching for genes that are differentially regulated in a region of embryonic cat brain called the lateral geniculate nucleus (LGN). They looked specifically between days 42 and 52 of gestation, when retinal input from the two eyes segregates into eye-specific zones. This activity-dependent repatterning of synaptic connectivity is blocked by tetrodotoxin, so the authors compared transcription in the LGN in the presence and absence of this toxin. They found just one messenger RNA that was differentially expressed, and this turned out, most surprisingly, to be the product of a known feline class I MHC gene.

Class I molecules usually present peptides derived from intracellular pathogens (such as viruses and bacteria) to the immune system. In the brain, most normal, resting cells have vanishingly low levels of MHC class I (ref. 2). Now, however, Shatz and colleagues show, through elegant in situ hybridizations using feline class I MHC probes, that spatio-temporal expression of both the mRNA and protein correlates closely with synaptic remodelling in the development of binocular vision.

Shatz et al. demonstrate that expression of class I MHC within the LGN is highest in the late fetal and early neonatal stages of development. This period is well after axons from the retina first reach the LGN, but is when their terminal processes segregate into eye-specific zones and their morphology is fine-tuned (Fig. 1a, overleaf). Segregation and fine-tuning depend on neuronal activity ${ }^{3,4}$, but it is the pattern rather than the level of activity that is important. In the late fetal period, populations of retinal ganglion cells spontaneously fire short bursts of action potentials and then remain silent $t^{5}$. Although neurons in the same place tend to fire at the same time within each retina, there is no correlation between firing patterns in the two eyes. Tetrodotoxin blocks the eventual segregation of inputs from the two eyes in the brain, so this simultaneous firing seems to be a kind of link among the ganglion cells of each eye. Thus, if 'neurons that fire together, wire together', the pattern of spontaneous activity will ensure segregation of input from the two eyes in the LGN. 